\title{
Compton thick AGN in Chandra sureys
}

\section{Murray Brightman and Kirpal Nandra}

\author{
Max-Planck-Institut für extraterrestrische Physik, Giessenbachstrasse 1, D-85748, Garching \\ bei München, Germany \\ email: mbright@mpe.mpg.de
}

\begin{abstract}
We present the results from the X-ray spectral analysis of active galactic nuclei (AGN) in the Chandra Deep Field-South, AEGIS-XD and Chandra-COSMOS surveys, focussing on the identification and characterisation of the most heavily obscured, Compton thick (CT, $N_{\mathrm{H}}>10^{24} \mathrm{~cm}^{-2}$ ) sources. Our sample is comprised of 3088 X-ray selected sources, which has a high rate of redshift completeness $(97 \%)$. The aim is to produce the largest and cleanest uniform sample of these sources from the data as possible. We identify these sources through X-ray spectral fitting, utilising torus spectral models designed for heavily obscured AGN which self consistently include the spectral signatures of heavy absorption, being Compton scattering, photoelectric absorption and iron K $\alpha$ fluorescence. We identify a total of 163 CT AGN covering an intrinsic 2-10 keV X-ray luminosity range of $10^{42}-3 \times 10^{45} \mathrm{erg} \mathrm{s}^{-1}$ and from $\mathrm{z}=0.1-7$.
\end{abstract}

\section{Introduction}

Understanding AGN obscuration is important for understanding the accretion history of the Universe (Fabian \& Iwasawa 1999) and for modelling the Cosmic X-ray background (CXB, Comastri et al. 1995). The most extreme examples of obscured AGN are so called Compton thick AGN, which are the least well understood. CTAGN are predicted to be a key phase in the growth of supermassive black holes (SMBH), where major mergers of galaxies cause the infall of gas to the nucleus, driving both black hole growth and growth of the galactic bulge (e.g. Hopkins et al. 2006). Understanding this phase has been challenging so far due to the relatively low numbers identified in X-ray surveys to date (e.g. Tozzi et al. 2006; Georgantopoulos et al. 2009; Comastri et al. 2011; Brightman \& Ueda 2012). The aim of this work is to undertake a systematic X-ray spectral analysis across three major extra-galactic Chandra survey fields survey fields in order to identify a large sample of CTAGN such that their cosmological significance may be assessed.

\section{Sample and Method}

Our sample consists of 3088 sources from the Chandra Deep Field South (4 Ms, Xue et al. 2011), AEGIS-XD (800 ks, Nandra et al. In preparation) and Chandra-COSMOS (160 ks, Elvis et al. 2009; Civano et al. 2012) surveys, which have a high rate of redshift completeness $(97 \%)$.

We utilise X-ray spectral torus models of Brightman \& Nandra (2011) which self consistently account for all signatures of obscuration, being photo-electric absorption, Compton scattering and $\mathrm{Fe} \mathrm{K} \alpha$ fluorescence, with the assumption of a torus geometry. The model also has a variable opening angle which allows us to test different scenarios for the covering factor of the obscuring material. Opening angles explored are $60^{\circ}, 30^{\circ}$ and $0^{\circ}$, where the latter represents the scenario where the source is completely covered in material. We also importantly include a secondary power-law component to model intrinsic emission that has been scattered into the line of sight by hot electrons filling the cone of the torus, for opening angles $>0^{\circ}$ (Turner et al. 1997). 


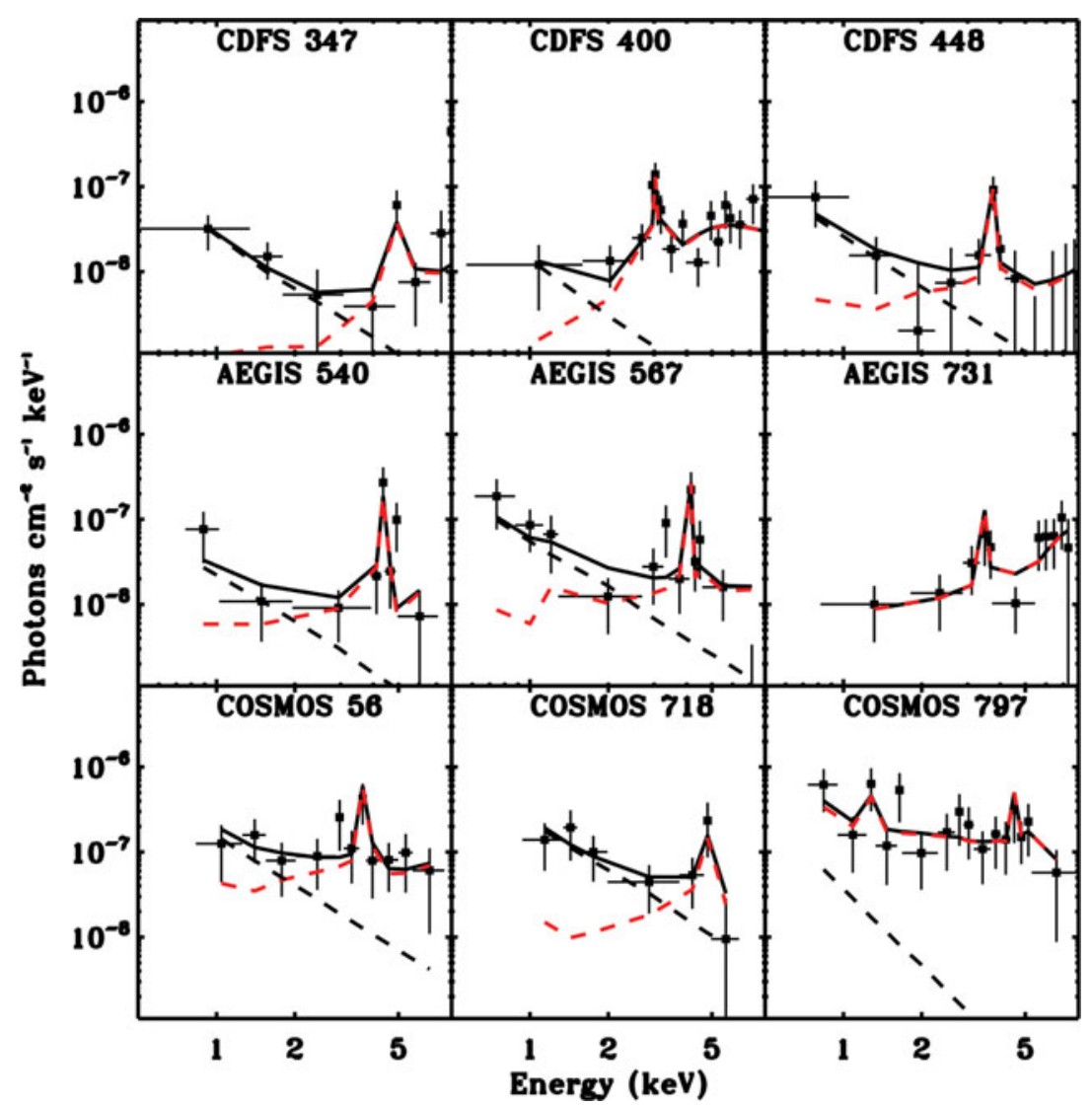

Figure 1. Example spectra of 9 Compton thick AGN identified in this work, three from each survey used. Red dashed lines show the torus model which represents the heavily obscured primary emission, whereas dashed black lines show the secondary power-law component, representing intrinsic scattered emission.

\section{Results}

Through our spectral analysis, we have identified a total of 163 CTAGN over a luminosity range of $10^{42}-3 \times 10^{45} \mathrm{erg} \mathrm{s}^{-1}$ and from $\mathrm{z}=0.1-7$, the largest sample of X-ray spectral CTAGN identifications to date. Of these, 37 were found in CDFS, 62 in AEGIS and 64 in COSMOS. Figure 1 below presents 9 example spectra from these sources.

\section{References}

Brightman M. \& Nandra K. 2011, MNRAS 413, 1206

Brightman M. \& Ueda Y., 2012, MNRAS 423, 702

Civano F., et al. 2012, ApJS 201, 30

Comastri A., et al. 2011, A\&SA 526, L9+

Elvis M., et al. 2009, ApJS 184, 158

Fabian A. C. \& Iwasawa K., 1999, MNRAS 303, L34

Georgantopoulos I., et al. 2009, A\&A 507, 747

Hopkins P. F., et al. 2006, ApJS 163, 1

Tozzi P., et al. 2006, A\&A 451, 457

Turner T. J., et al. 1997, ApJS 113, 23

Xue Y. Q., et al. 2011, ApJS 195, 10 